\title{
RECONSTUCTION WITH COMPUTERIZED MICROWAVE DIFFRACTION TOMOGRAPHY BY USING CIRCULAR MEASUREMENT SYSTEM IN THE FAR-FIELD REGION
}

\author{
Sedef KENT Bingül YAZGAN \\ Istanbul Technical University, Electrical \& Electronics Engineering Faculty, 80626, Maslak \\ Istanbul
}

Abstract- This paper deals with far-field microwave imaging of a dielectric object satisfying the Born approximation. The results of computer simulations obtained with a circular measuring geometry are given. The computational efficiency of this method is also shown.

\section{INTRODUCTION}

The field of computed tomography involves the nondestructive reconstruction of a slice of an object from measurement made external to the object. There are a variety of ways in which the object is irradiated and in which the measurements are made. Electromagnetic waves are diffracted and refracted when they encounter scattering objects. For this reason this type of tomography is named diffraction tomography. According to the Fourier diffraction projection theorem [1]-[2], by illuminating an object in many different directions and measuring the diffracted projection data, one can fill up the frequency domain with the samples of the object over circular arcs and then reconstruct the object by Fourier inversion. This theorem is valid when the inhomogeneties in the object are weakly scattering.

Electromagnetic diffraction tomography is the inversion of scattered waves from an object, based on the Helmholtz wave equation. For the case of weak scattering, the first order Born approximation gives the relation between the object function and the scattered field as [1]

$E^{s}(\mathbf{k}, \mathbf{r})=\mathbf{k}^{2} \int \mathrm{G}\left(\mathbf{r}-\mathbf{r}^{\prime}\right) \mathrm{O}\left(\mathbf{r}^{\prime}\right) \mathrm{E}^{\mathrm{i}}\left(\mathrm{k}, \mathbf{r}^{\prime}\right) \mathrm{d}^{2} \mathbf{r}^{\prime}$

where $k$ is the wavenumber, $\mathrm{G}($.$) is the free space Green's fuction, \mathrm{O}()$ is the object function given by

$O(\mathbf{r})=\varepsilon(\mathbf{r})-1$

$\mathrm{E}^{\mathrm{i}}$ and $\mathrm{E}^{\mathrm{s}}$ are the incident and scattered fields, respectively. The boldface letters are used for vector representation. In the two dimensional case the Green's function is written as

$G\left(r-r^{\prime}\right)=-(j / 4) H_{o}^{(2)}\left(k\left|r-r^{\prime}\right|\right)$ 
where $\mathrm{H}_{0}{ }^{(2)}($.$) represents the Hankel function of the second kind and order zero.$ Reconstructions can only be accurately obtained when the object under examination is a weakly scattering object.

\section{FORMULATION OF THE PRORLEM}

An infinite dielectric cylinder whose axis is taken parallel with the z-axis of a rectangular coordinate system is considered. The incident plane wave, propagating in the direction of the unit vector $s_{0}$, which is normal to the $\mathrm{z}$-axis is given by

$E^{i}\left(k, r^{\prime}\right)=\exp \left(-j k s_{0} \cdot r\right)$

where $\mathbf{k}$ is the wavenumber of the surrounding medium, $\mathbf{r}$ is the position vector and $\exp (+j \omega t)$ is implied. The scattered fields from the object can be measured by a circular antenna array at a constant distance $|\mathbf{r}|$ from the center of the object as shown in Fig. 1 . If we assume that the observation point is in the far field, the distance from the scatterer to the receiver point can be simplified as

$R=\left|r-r^{9}\right|=\left(r^{2}+r^{\prime 2}-2 r \cdot r^{9} \cdot(-s)\right)^{1 / 2} \approx r+r^{\prime} \cdot \mathbb{S}$

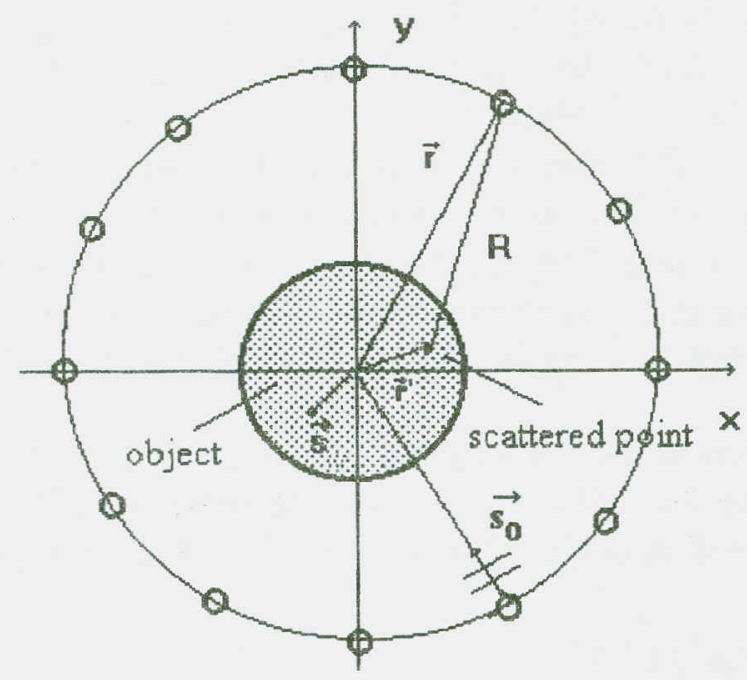

Fig. 1- Circular measuring geometry

where $s$ is the unit vector in the opposite direction of $r$. By using the incident plane waves as in (3) and the Hankel function representation of the Green's function in (1) the scattered field is obtained as

$E^{s}(k, r)=\left(j k^{2} / 4\right) \int G\left(r-r^{\prime}\right) O\left(r^{\prime}\right) E^{i}\left(k, r^{\prime}\right) d^{2} r^{\prime}$ 
Since we assumed that the receiver is in the far field region, the asymptotic expansion of the Hankel function can be used to obtain,

$\mathrm{E}^{S}(\mathrm{k}, \mathrm{r}) \approx-\mathrm{j} \sqrt{\frac{k^{3}}{8 \pi r}} e^{j \pi / 4} e^{-j k r} \int O\left(\bar{r}^{\prime}\right) e^{-j k\left(\bar{s}+\bar{s}_{o}\right)} d^{2} r$

The integral term in (7) represents the two-dimensional (2-D) Fourier transformation of the object function $O(\mathbf{r})$. In this situation, the relation between the far field scattered wave and the 2-D Fourier transform of the object can be obtained as

$\mathrm{E}^{\mathrm{S}}\left(\mathrm{k}, \mathrm{r}_{\mathrm{r}}\right) \approx-j k^{3 / 2} e^{j \pi / 4} \frac{e^{-j k r}}{\sqrt{8 \pi r}} \bar{O}\left(k\left(\bar{s}+\bar{s}_{o}\right)\right)$

Since the distance $\mathrm{r}$ is constant in the proposed circular antenna array, the multiplication terms in (8) yield a constant factor. To obtain the $O(x, y)$ distribution of the object in Cartesian coordinate system, by inverse transformation, first the location of the $\mathrm{O}\left(\mathrm{k}\left(\mathrm{s}+\mathrm{s}_{\mathrm{o}}\right)\right)$ in the $O(u, v)$ frequency domain must be found.

In the circular antenna array every antenna element in its turn, is considered to be the transmitter, while the others are receivers. Let the angle between the $s_{0}$ and the horizontal axis be $\mathbf{X}_{\mathrm{o}}$. Similarly, let the angle between the antenna position vector $\mathbf{s}$ and the horizontal axis be $X$. The known values of the Fourier transform $O\left(k\left(s+s_{0}\right)\right)$ of the object function are on a circle as shown in Fig. 2 . This circle has its center at $\mathbf{k s}_{\mathrm{o}}$ and its radius is $\mathrm{k}$. When the

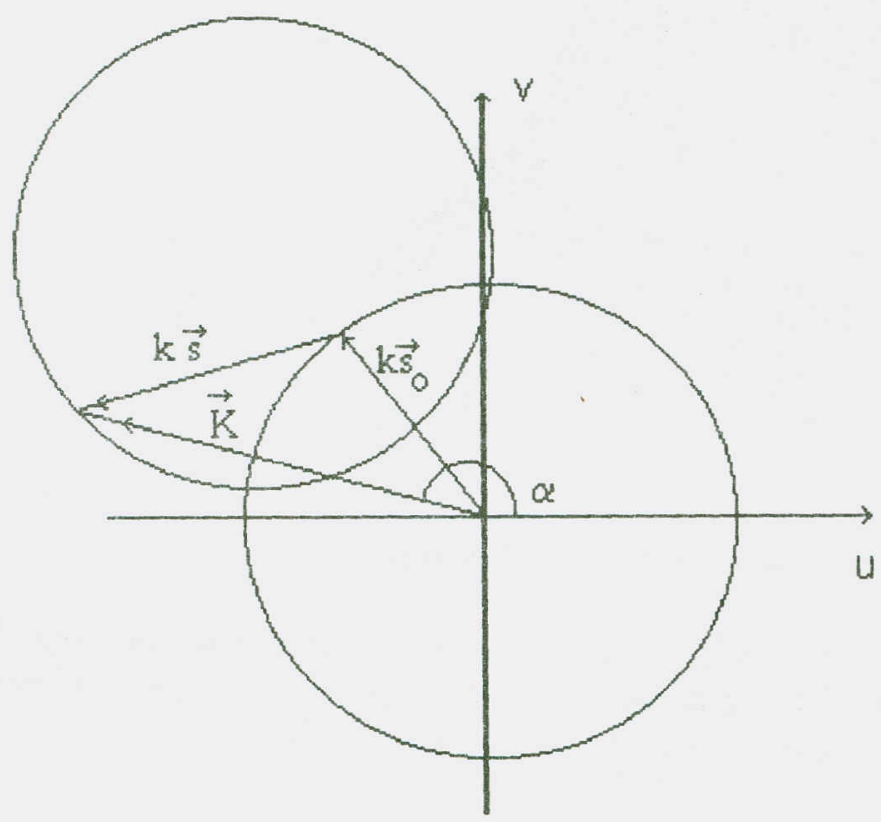

Fig.2-Location of circles in the frequency domain. 
angle $\mathrm{X}$ changes with the antenna receiver element, the circle also changes. A point on the circle can be shown with the vector $\mathbf{K}$ given by

$$
\bar{K}=k \bar{s}_{o}+k \bar{s}=|\bar{K}| e^{j \alpha}
$$

As a result, each set of data obtained when one of the antenna element transmit while others receive results in a circle in the frequency plane of diameter $2 k$. This case will improve the image quality. If there are $\mathrm{N}$ antenna elements, the received field values are measured at all the elements for each element used as the transmitter. This means the field values are converted into the frequency domain. The resulting matrix $Q(K, \alpha)$ is a $2 \mathrm{Nx} 2 \mathrm{~N}$ matrix [2]. To obtain the object function on a rectangular grid as required by the 2-D inverse FFT algorithm, the nearest-neighbor interpolation technique is used. By taking the 2-D inverse FFT of the resulting $O(u, v)$ matrix, the object function $O(x, y)$ is obtained.

\section{NUMERICAL RESULTS}

The reconstruction of a cylinder of $0.06 \mathrm{~m}$ in radius with a permittivity of 1.035 is given in Fig. 3. 32 antenna elements are used in the measuring system and the radius of the circular measuring geometry is 20 wavelengths.

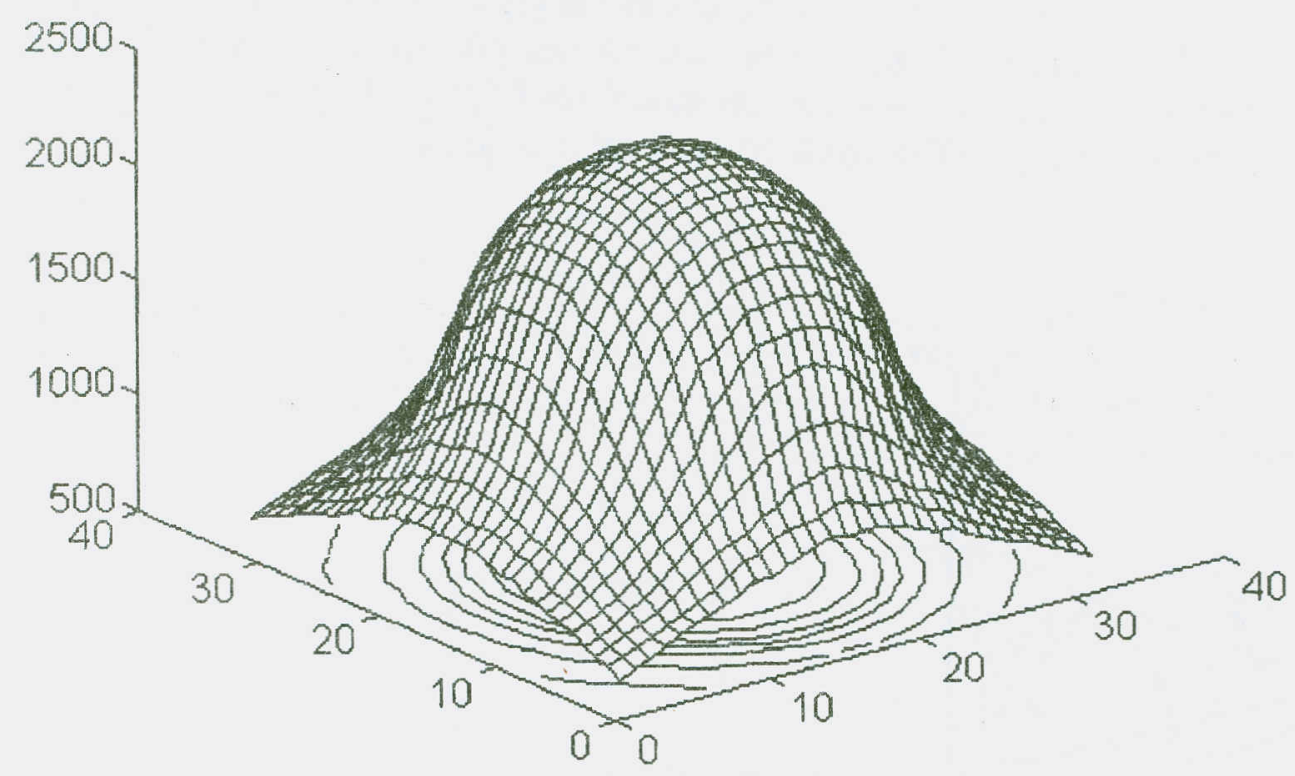

Fig.3- The reconstruction of a cylinder obtained by 32 antennas.

Another reconstruction is given in Fig.4. Here, a three-layered cylindrical object is reconstructed by using 32 antennas. The diameters of the layers are $r_{1}=0.2 \mathrm{~m}, r_{2}=0.3 \mathrm{~m}$ and $\mathrm{r}_{3}=0.4 \mathrm{~m}$ and the dielectric permittivities are $\varepsilon_{1}=1.3, \varepsilon_{2}=1.0$, and $\varepsilon_{3}=1.8$. 
The comparison of these results with the other methods can be found in [3]. Here, it can be seen that the Mean Squared Error (MSE) will decrease by using of circular geometry in the far-field region, because the reached area in the frequency domain has a radius of $2 \mathrm{k}$.

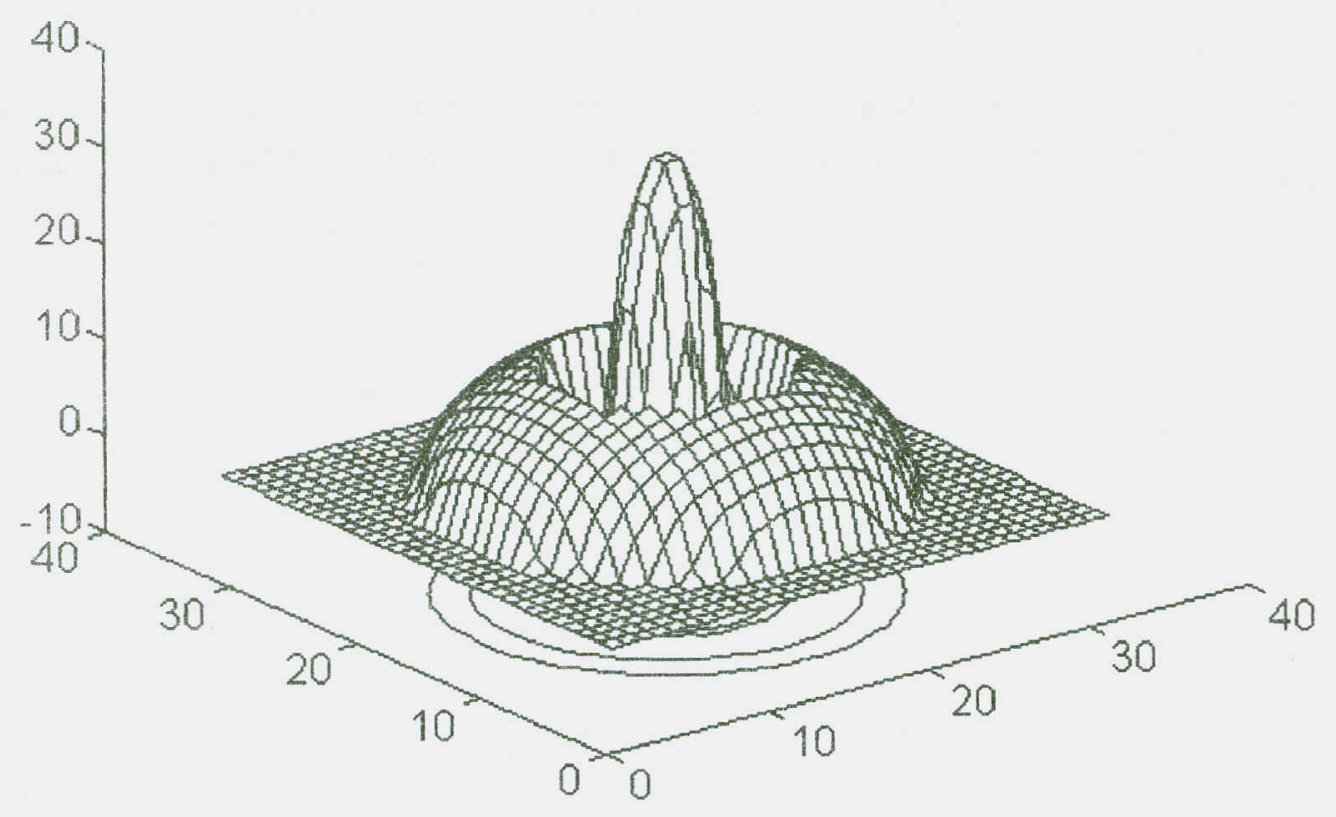

Fig.4- The reconstruction of a three-layered cylinder obtained by 32 antennas

An FFT takes NLogN operations. For the algorithm using Fourier diffraction projection theorem, it is needed 3(NLogN) operations. But in the far-field region 2(NLogN) operations are sufficient.

\section{CONCLUSION}

A reconstruction method with computerized microwave diffraction tomography by using circular measurement system in the far-field region is presented. To image of a dielectric object the first order Born approximation is used. The results of computer simulations obtained with a circular measuring geometry are given. This method improves the image quality because the size of the area reached in the frequency domain becomes $2 k$ instead of $\mathrm{k}$ as in the near field tomography systems. For this reason, the reconstructions are better than the others even though the same numbers of projections and samples were used. There is no need to rotate the transmitter- receiver system or the object itself. Computational time is also reduced because it is not necessary to take 1-D Fourier transform of the projections before locate them in the frequency domain.

\section{RETERENCES}

[1] SLANEY, M., KAK, A.C., LAERSEN, L.E., "Limitation of imaging with first order diffraction tomography", IEEE Trans. Microwave Theory Tech.. MTT-32, pp.860-873, 1984. 
[2] PAN, S.X:, KAK, A.C.," A computational study of reconstruction algorithms for diffraction tomography: Interpolation vs. Filtered backpropagation" IEEE Trans. Acoust. Speech Signal Processing, Vol. ASSP-31, pp.1262-1275, 1983.

[3] KENT, S. "A new algorithm for microwave diffraction tomography and its comparison with the other reconstruction methods" Ph.D. Thesis, Istanbul Technical University, December 1989. 